\title{
The Geometry of Tangent Bundles: Canonical Vector Fields
}

\author{
Tongzhu Li ${ }^{1}$ and Demeter Krupka ${ }^{1,2,3}$ \\ ${ }^{1}$ Department of Mathematics, Beijing Institute of Technology, Beijing 100081, China \\ ${ }^{2}$ Department of Mathematics, Faculty of Science, The University of Ostrava, 30. dubna 22, 70103 Ostrava, Czech Republic \\ ${ }^{3}$ Department of Mathematics, La Trobe University, Melbourne, Bundoora, VIC 3086, Australia
}

Correspondence should be addressed to Demeter Krupka; demeter.krupka@lepageri.eu

Received 14 December 2012; Accepted 13 March 2013

Academic Editor: Anna Fino

Copyright (C) 2013 T. Li and D. Krupka. This is an open access article distributed under the Creative Commons Attribution License, which permits unrestricted use, distribution, and reproduction in any medium, provided the original work is properly cited.

\begin{abstract}
A canonical vector field on the tangent bundle is a vector field defined by an invariant coordinate construction. In this paper, a complete classification of canonical vector fields on tangent bundles, depending on vector fields defined on their bases, is obtained. It is shown that every canonical vector field is a linear combination with constant coefficients of three vector fields: the variational vector field (canonical lift), the Liouville vector field, and the vertical lift of a vector field on the base of the tangent bundle.
\end{abstract}

\section{Introduction}

Vector fields on tangent bundles belong to basic concepts of pure and applied differential geometry, global analysis, and mathematical physics. Recent research in geometry extends the well-known correspondence of semisprays, sprays, and geodesic sprays to the classical theory of geodesics and connections (see, e.g., $[1,2])$. Vector fields on tangent bundles can be considered as an underlying geometric structure for the theory of second-order differential equations [37]. The semispray theory has been used in the calculus of variations on manifolds to characterize extremal curves of a variational functional as integral curves of the Hamilton or Euler-Lagrange vector fields $[2,4,8,9]$. Sprays and semisprays also provide a natural framework for extension of classical results of analytical mechanics to contemporary mechanical problems and stimulate a broad research in the global theory of nonconservative systems, symmetries, and the constraint theory (see, e.g., $[6,10-12])$.

This paper is devoted to the structure theory of vector fields on the tangent bundle $T X$ of a manifold $X$; our aim will be to classify all canonical vector fields on $T X$, independent of any geometric structure or on the topology of $X$. Our main theorem says that every canonical vector field is a linear combination with constant coefficients of three independent vector fields: (a) a variational vector field (the natural lift of a vector field, defined on $X$ ), (b) the Liouville vector field, and (c) the vertical lift of a vector field on $X$; this completes the results obtained in [13]. Another result is the method how the main theorem has been formulated and proved; the concepts we use allow generalizations and applications to analogous problems of discovering and describing canonical geometric objects.

Our approach to the problem is based on the theory of jets and differential invariants, and on an observation that the coordinate transformations on $X$ naturally define a Lie group $L_{n}^{r}$, where $n=\operatorname{dim} X$, the differential group, and its left action on the type fibre of any natural bundle over $X[13-$ 17]. The general theory gives us equations that determine a canonical vector field on $T X$ as a differential invariant of the second differential group $L_{n}^{r}$.

The proof of our result is not straightforward; it relies on the semidirect product structure of $L_{n}^{r}$ and on the orbit reduction method that has already been applied to the classification problem of differential invariants of a linear connection [18]. The method can also be used in the canonical constructions depending on any geometric objects.

Throughout this work, $X$ is a smooth real $n$-dimensional manifold, TX is the tangent bundle of $X$, and $\tau_{X}: T X \rightarrow X$ is the tangent bundle projection. The second tangent bundle of $X$ is the tangent bundle TTX $=T^{2} X$ over TX with the tangent bundle projection $\tau_{T X}: T T X \rightarrow T X$; its elements are second-order tangent vectors on $X$. The mapping $T \tau_{X}$ is the differential $T \tau_{X}: T T X \rightarrow T x$ of $\tau_{X}$, satisfying 
$\tau_{X} \circ T \tau_{x}=\tau_{X} \circ \tau_{T X}$. The 1-jet prolongation of $T X$ is denoted by $J^{1} T X$; elements of the set $J^{1} T X$ are the 1-jets $J_{x}^{1} \xi$ with source $x \in X$ and target $\xi(x) \in T_{x} X=\tau_{x}^{-1}(x)$; the source and target jet projections are $\tau_{X}^{1}: J^{1} T X \rightarrow X$ and $\tau_{X}^{1,0}: J^{1} T X \rightarrow T X$.

\section{Second-Order Vectors and Jets of a Vector Field}

In this section, we fix basic notation, used throughout this paper. If $(U, \varphi), \varphi=\left(x^{i}\right)$, is a chart on $X$, we denote by $\left(\tau_{X}^{-1}(U), \Phi\right), \Phi=\left(x^{i}, \dot{x}^{i}\right)$, the associated chart on the tangent bundle $T X$; the associated chart on $T^{2} X$ is denoted by $\left(\tau_{T X}^{-1}\left(\tau_{X}^{-1}(U)\right), \Psi\right), \Psi=\left(x^{i}, \dot{x}^{i}, \widetilde{x}^{i}, \widetilde{x}^{i}\right)$. The tangent bundle projection $\tau_{X}$ has the chart expression $\left(x^{i}, \dot{x}^{i}\right) \rightarrow\left(x^{i}\right)$, and $\tau_{T X}$ is expressed by $\left(x^{i}, \dot{x}^{i}, \tilde{x}^{i}, \tilde{x}^{i}\right) \rightarrow\left(x^{i}, \dot{x}^{i}\right)$. The tangent mapping $T \tau_{X}$ is expressed in coordinates as $\left(x^{i}, \dot{x}^{i}, \widetilde{x}^{i}, \tilde{\dot{x}}^{i}\right) \rightarrow$ $\left(x^{i}, \widetilde{x}^{i}\right)$.

In the following two lemmas, we recall standard transformation formulas, needed in proofs.

Lemma 1. Let a second-order tangent vector $\Xi \in T_{\zeta} T X$ be expressed in two charts $(U, \varphi), \varphi=\left(x^{i}\right)$, and $(V, \psi), \psi=\left(y^{i}\right)$, as

$$
\Xi=\Xi^{k}\left(\frac{\partial}{\partial x^{k}}\right)_{\zeta}+\dot{\Xi}^{k}\left(\frac{\partial}{\partial \dot{x}^{k}}\right)_{\zeta}=\Theta^{l}\left(\frac{\partial}{\partial y^{l}}\right)_{\zeta}+\dot{\Theta}^{l}\left(\frac{\partial}{\partial \dot{y}^{l}}\right)_{\zeta}
$$

Then,

$$
\begin{gathered}
\Theta^{l}=\left(\frac{\partial y^{l}}{\partial x^{k}}\right)_{\varphi(x)} \Xi^{k}, \\
\dot{\Theta}^{l}=\left(\frac{\partial^{2} y^{l}}{\partial x^{p} \partial x^{k}}\right)_{\varphi(x)} \dot{x}^{p}(\zeta) \Xi^{k}+\left(\frac{\partial y^{l}}{\partial x^{k}}\right)_{\varphi(x)} \dot{\Xi}^{k} .
\end{gathered}
$$

The chart on $J^{1} T X$, associated with the chart $(U, \varphi), \varphi=$ $\left(x^{i}\right)$, on $X$ is denoted by $\left(\left(\tau_{x}^{1}\right)^{-1}(U), \Phi^{1}\right), \Phi^{1}=\left(x^{i}, \dot{x}^{i}, \dot{x}_{j}^{i}\right)$. The coordinates of a 1-jet $J_{x}^{1} \xi$ are $x^{i}\left(J_{x}^{1} \xi\right)=x^{i}(x), \dot{x}^{i}\left(J_{x}^{1} \xi\right)=$ $\dot{x}^{i}(\xi(x))$, and $\dot{x}_{j}^{i}\left(J_{x}^{1} \xi\right)=D_{j}\left(\dot{x}^{i} \xi \varphi^{-1}\right)(\varphi(x))$.

Lemma 2. For any two charts $(U, \varphi), \varphi=\left(x^{i}\right)$, and $(V, \psi), \psi=$ $\left(y^{i}\right)$, on $X$, such that $U \cap V \neq \emptyset$, the transformation equations on $\left(\tau_{X}^{1}\right)^{-1}(U) \cap\left(\tau_{X}^{1}\right)^{-1}(U)$ are

$$
\dot{y}^{p}=\frac{\partial y^{p}}{\partial x^{m}} \dot{x}^{m}, \quad \dot{y}_{q}^{p}=\left(\frac{\partial^{2} y^{p}}{\partial x^{s} \partial x^{m}} \dot{x}^{m}+\frac{\partial y^{p}}{\partial x^{m}} \dot{x}_{s}^{m}\right) \frac{\partial x^{s}}{\partial y^{q}} .
$$

We need the pullback fibration $\tau_{X}^{*} J^{1} T X=\left\{\left(\zeta, J_{x}^{1} \xi\right) \epsilon\right.$ $\left.T X \times J^{1} T X \mid \tau_{X}(\zeta)=x\right\}$ over $T X$. We have the commutative diagram

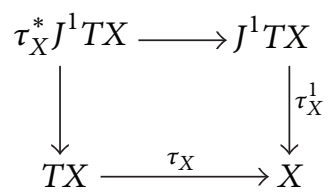

in which the left vertical arrow is the restriction of the first Cartesian projection $p r_{1}: T X \times J^{1} T X \rightarrow T X$ and the upper horizontal arrow is the restriction of the second Cartesian projection $p r_{2}: T X \times J^{1} T X \rightarrow J^{1} T X$. We denote

$$
\rho_{X}=p r_{1} .
$$

Any chart $(U, \varphi), \varphi=\left(x^{i}\right)$, on $X$ induces a chart on the pullback manifold $\tau_{X}^{*} J^{1} T X$. Denoting by $x^{i}(\zeta)=x^{i}(x), \dot{x}^{i}(\zeta)=\zeta^{i}$ the coordinates of a vector $\zeta \in \tau_{X}^{-1}(U)$ at $x \in U$ and by $x^{i}\left(J_{x}^{1} \xi\right)=x^{i}(x), \dot{x}^{i}\left(J_{x}^{1} \xi\right)=\xi^{i}, \dot{x}_{j}^{i}\left(J_{x}^{1} \xi\right)=\xi_{j}^{i}$ the coordinates of a 1-jet $J_{x}^{1} \xi \in\left(\tau_{X}^{1}\right)^{-1}(U)$, then the induced chart on $\tau_{X}^{*} J^{1} T X$, $\left(\rho_{x}^{-1}\left(\tau_{X}^{-1}(U)\right), \widetilde{\Phi}^{1}\right), \widetilde{\Phi}^{1}=\left(x^{i}, \dot{x}^{i}, \dot{z}^{i}, \dot{z}_{j}^{i}\right)$, is defined by

$$
\begin{gathered}
x^{i}\left(\zeta, J_{x}^{1} \xi\right)=x^{i}(x), \quad \dot{x}^{i}\left(\zeta, J_{x}^{1} \xi\right)=\zeta^{i}, \\
\dot{z}^{i}\left(\zeta, J_{x}^{1} \xi\right)=\xi^{i}, \quad \dot{z}_{j}^{i}\left(\zeta, J_{x}^{1} \xi\right)=\xi_{j}^{i} .
\end{gathered}
$$

In these coordinates, $\rho_{X}$ is the mapping $\left(x^{i}, \dot{x}^{i}, \dot{z}^{i}, \dot{z}_{j}^{i}\right) \rightarrow$ $\left(x^{i}, \dot{x}^{i}\right)$ and the second Cartesian projection is $\left(x^{i}, \dot{x}^{i}, \dot{z}^{i}, \dot{z}_{j}^{i}\right) \rightarrow\left(x^{i}, \dot{x}^{i}, \dot{z}_{j}^{i}\right)$.

\section{Differential Groups and Differential Invariants}

Recall that for any positive integer $r$, the $r$ th differential group $L_{n}^{r}$ is the group of invertible $r$-jets with source and target at the origin 0 , endowed with its natural Lie group structure; the group multiplication in $L_{n}^{r}$ is the jet composition $L_{n}^{r} \times L_{n}^{r}$ э $J_{0}^{r} \alpha \circ J_{0}^{r} \beta=J_{0}^{r}(\alpha \circ \beta) \in L_{n}^{r}$. The first differential group $L_{n}^{1}$ is just the group $G L_{n}(\mathbf{R})$. For all $r \geq s$, we denote by $\pi^{r, s}: L_{n}^{r} \rightarrow L_{n}^{s}$ the canonical jet projection and by $\iota^{1, r}$ : $L_{n}^{1} \rightarrow L_{n}^{r}$ the canonical injective Lie group morphism. The normal subgroup $K_{n}^{r}=\operatorname{Ker} \pi^{r, 1}$ of $L_{n}^{r}$ is nilpotent, and $L_{n}^{r}$ is the interior semidirect product of $\iota^{1, r}\left(L_{n}^{1}\right)$ and $K_{n}^{r}[13,17]$; we denote $L_{n}^{r}=L_{n}^{1} \times{ }_{s} K_{n}^{r}$.

The first canonical coordinates on $L_{n}^{r}$ are the functions $a_{j_{1}}^{i}, a_{j_{1} j_{2}}^{i}, \ldots, a_{j_{1} j_{2} \cdots j_{r}}^{i}$, where $1 \leq j_{1} \leq j_{2} \leq \cdots \leq j_{k} \leq n$, $k=1,2, \ldots, r$, defined as follows. If $J_{0}^{r} \alpha \in L_{n}^{r}$ is an $r$ jet and $\alpha=\left(\alpha^{i}\right)$ its representative, then $a_{j_{1} j_{2} \cdots j_{k}}^{i}\left(J_{0}^{r} \alpha\right)=$ $D_{j_{1}} D_{j_{2}} \cdots D_{j_{k}} \alpha^{i}(0)$. Similarly, the formula $b_{j_{1} j_{2} \cdots j_{k}}^{i}\left(J_{0}^{r} \alpha\right)=$ $a_{j_{1} j_{2} \cdots j_{k}}^{i}\left(J_{0}^{r} \alpha^{-1}\right)$ defines the second canonical coordinates $b_{j_{1}}^{i}, b_{j_{1} j_{2}}^{i}, \ldots, b_{j_{1} j_{2} \cdots j_{r}}^{i} ;$ clearly, these coordinates satisfy

$$
a_{j}^{i} b_{k}^{j}=\delta_{k}^{i} .
$$

Equations of the subgroup $K_{n}^{r}$ are $a_{j}^{i}=\delta_{j}^{i}$, and equations of the subgroup $\iota^{1, r}\left(L_{n}^{1}\right)$ are $a_{j_{1} j_{2}}^{i}=0, \ldots, a_{j_{1} j_{2} \cdots j_{r}}^{i}=0$.

By a differential invariant, we mean an $L_{n}^{r}$-equivariant mapping $f: P \rightarrow Q$ of left $L_{n}^{r}$-manifolds [13]. Given the actions of $L_{n}^{r}$ on $P$ and $Q$, we get the equation for the differential invariant $f$ :

$$
f\left(J_{0}^{r} \alpha \cdot p\right)=J_{0}^{r} \alpha \cdot f(p)
$$


where $p \in P$ and $J_{0}^{r} \alpha \in L_{n}^{r}$. Equation (8) splits to an equivalent system:

$$
f\left(J_{0}^{r} \alpha \cdot p\right)=J_{0}^{r} \alpha \cdot f(p), \quad f\left(J_{0}^{r} \beta \cdot p\right)=J_{0}^{r} \beta \cdot f(p),
$$

where $J_{0}^{r} \alpha \in L_{n}^{1}$ and $J_{0}^{r} \beta \in K_{n}^{r}$ (the orbit reduction method [18]).

The problem we consider in this paper reduces to solving (9) for some specific left $L_{n}^{2}$-manifolds $P$ and $Q$. In our case, the orbit reduction method simplifies (8) and allows us to obtain its complete solution.

\section{Canonical Vector Fields on Tangent Bundles}

Any diffeomorphism $\alpha: U \rightarrow X$, defined on an open set $U$ in $X$, induces the corresponding lifted diffeomorphisms $T \alpha: T U \rightarrow T X, J^{1} T \alpha: J^{1} T U \rightarrow J^{1} T X$, and $\left(T \alpha, J^{1} T \alpha\right):$ $\tau_{X}^{*} J^{1} T U \rightarrow \tau_{X}^{*} J^{1} T X$. By a canonical vector field on $T X$, we mean a morphism $\Xi: \tau_{X}^{*} J^{1} T X \rightarrow T^{2} X$ such that

$$
\tau_{T X} \circ \Xi=\rho_{X},
$$

and for all diffeomorphisms $\alpha: U \rightarrow X$ of $X$,

$$
\Xi \circ\left(T \alpha, J^{1} T \alpha\right)=T(T \alpha) \circ \Xi .
$$

Condition (10) means that the diagram

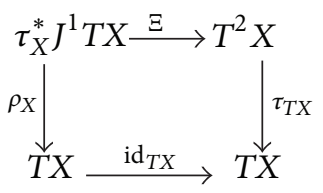

commutes or, which is the same, for all $\left(\zeta, J_{x}^{1} \xi\right) \in \tau_{X}^{*} J^{1} T X$, $\Xi\left(\zeta, J_{x}^{1} \xi\right)$ is a second-order vector on $X$ at the point $\zeta \in T X$,

$$
\tau_{T X}\left(\Xi\left(\zeta, J_{x}^{1} \xi\right)\right)=\zeta
$$

Since $J^{1} T \alpha\left(J_{x}^{1} \xi\right)=J_{\alpha(x)}^{1}\left(T \alpha \circ \xi \circ \alpha^{-1}\right)$, condition (11) can also be written as

$$
\Xi\left(T_{x} \alpha \cdot \zeta, J_{\alpha(x)}^{1}\left(T \alpha \circ \xi \circ \alpha^{-1}\right)\right)=T_{\zeta} T \alpha \cdot \Xi\left(\zeta, J_{x}^{1} \xi\right) .
$$

We express conditions (13) and (14) in coordinates. We write in a chart $(U, \varphi), \varphi=\left(x^{i}\right)$,

$$
\Xi\left(\zeta, J_{x}^{1} \xi\right)=\Xi_{(U, \varphi)}^{k}\left(\zeta, J_{x}^{1} \xi\right)\left(\frac{\partial}{\partial x^{k}}\right)_{\zeta}+\dot{\Xi}_{(U, \varphi)}^{k}\left(\zeta, J_{x}^{1} \xi\right)\left(\frac{\partial}{\partial \dot{x}^{k}}\right)_{\zeta}
$$

Then for fixed $x$, the components are functions of the coordinates $\zeta^{i}, \xi^{i}$, and $\xi_{l}^{i}$; that is,

$$
\Xi_{(U, \varphi)}^{k}=\Xi_{(U, \varphi)}^{k}\left(\zeta^{i}, \xi^{i}, \xi_{l}^{i}\right), \quad \dot{\Xi}_{(U, \varphi)}^{k}=\dot{\Xi}_{(U, \varphi)}^{k}\left(\zeta^{i}, \xi^{i}, \xi_{l}^{i}\right) .
$$

Theorem 3. Let $\Xi: \tau_{X}^{*} J^{1} T X \rightarrow T^{2} X$ be a morphism over the identity $\mathrm{id}_{T X}$. The following two conditions are equivalent:
(1) $\Xi$ is a canonical vector field.

(2) For any points $x, y \in X$, any charts $(U, \varphi), \varphi=\left(x^{i}\right)$, at $x$ and $(V, \psi), \psi=\left(y^{i}\right)$, at $y$, and any diffeomorphism $\alpha: U \rightarrow V$ such that $\alpha(x)=y$,

$$
\begin{aligned}
& \Xi_{(V, \psi)}^{l}\left(a_{k}^{i} \zeta^{k}, a_{k}^{i} \xi^{k}, a_{k m}^{i} b_{j}^{m} \xi^{k}+a_{k}^{i} b_{j}^{m} \xi_{m}^{k}\right) \\
& =a_{k}^{l} \Xi_{(U, \varphi)}^{k}\left(\zeta^{i}, \xi^{i}, \xi_{j}^{i}\right), \\
& \dot{\Xi}_{(V, \psi)}^{l}\left(a_{k}^{i} \zeta^{k}, a_{k}^{i} \xi^{k}, a_{k m}^{i} b_{j}^{m} \xi^{k}+a_{k}^{i} b_{j}^{m} \xi_{m}^{k}\right) \\
& =a_{j k}^{l} \zeta^{j} \Xi_{(U, \varphi)}^{k}\left(\zeta^{i}, \xi^{i}, \xi_{m}^{i}\right)+a_{k}^{l} \dot{\Xi}_{(U, \varphi)}^{k}\left(\zeta^{i}, \xi^{i}, \xi_{m}^{i}\right),
\end{aligned}
$$

where

$$
\begin{gathered}
a_{k}^{l}=\left(\frac{\partial\left(y^{l} \alpha \varphi^{-1}\right)}{\partial x^{k}}\right)_{\varphi(x)}, \quad a_{j k}^{l}=\left(\frac{\partial^{2}\left(y^{l} \alpha \varphi^{-1}\right)}{\partial x^{j} \partial x^{k}}\right)_{\varphi(x)}, \\
b_{j}^{k}=\left(\frac{\partial\left(x^{k} \alpha^{-1} \psi^{-1}\right)}{\partial y^{j}}\right)_{\psi(x)} .
\end{gathered}
$$

Proof. Consider condition (14). With the abbreviations (18), the coordinates of the vector $T_{x} \alpha \cdot \zeta$ and the 1-jet $J_{\alpha(x)}^{1}(T \alpha \circ \xi \circ$ $\left.\alpha^{-1}\right)$ are

$$
\begin{gathered}
\dot{y}^{i}\left(T_{x} \alpha \cdot \zeta\right)=a_{k}^{i} \zeta^{k}, \\
\dot{y}^{i}\left(J_{\alpha(x)}^{1}\left(T \alpha \circ \xi \circ \alpha^{-1}\right)\right)=a_{k}^{i} \xi^{k}, \\
\dot{y}_{j}^{i}\left(J_{\alpha(x)}^{1}\left(T \alpha \circ \xi \circ \alpha^{-1}\right)\right)=a_{k l}^{i} b_{j}^{l} \xi^{k}+a_{k}^{i} b_{j}^{l} \xi_{l}^{k} .
\end{gathered}
$$

The coordinates of the vector $T_{\zeta} T \alpha \cdot \Xi\left(\zeta, J_{x}^{1} \xi\right)$ are determined by

$$
\begin{aligned}
T_{\zeta} T \alpha \cdot \Xi & \left(\zeta, J_{x}^{1} \xi\right) \\
= & a_{k}^{l} \Xi_{(U, \varphi)}^{k}\left(\zeta, J_{x}^{1} \xi\right)\left(\frac{\partial}{\partial y^{l}}\right)_{T_{x} \alpha \cdot \zeta} \\
& +\left(a_{k j}^{l} \dot{x}^{j}(\zeta) \Xi_{(U, \varphi)}^{k}\left(\zeta, J_{x}^{1} \xi\right)\right. \\
& \left.+a_{k}^{l} \dot{\Xi}_{(U, \varphi)}^{k}\left(\zeta, J_{x}^{1} \xi\right)\right)\left(\frac{\partial}{\partial \dot{y}^{l}}\right)_{T_{x} \alpha \cdot \zeta} .
\end{aligned}
$$

Writing

$$
\begin{aligned}
\Xi\left(T_{x} \alpha \cdot \zeta, J_{\alpha(x)}^{1}\left(T \alpha \circ \xi \circ \alpha^{-1}\right)\right) \\
=\Xi_{(V, \psi)}^{l}\left(T_{x} \alpha \cdot \zeta, J_{\alpha(x)}^{1}\left(T \alpha \circ \xi \circ \alpha^{-1}\right)\right)\left(\frac{\partial}{\partial y^{l}}\right)_{T_{x} \alpha \cdot \zeta} \\
\quad+\dot{\Xi}_{(V, \psi)}^{l}\left(T_{x} \alpha \cdot \zeta, J_{\alpha(x)}^{1}\left(T \alpha \circ \xi \circ \alpha^{-1}\right)\right)\left(\frac{\partial}{\partial \dot{y}^{l}}\right)_{T_{x} \alpha \cdot \zeta},
\end{aligned}
$$


we can express condition (14), with help of (19) and (20), as

$$
\begin{gathered}
\Xi_{(V, \psi)}^{l}\left(a_{k}^{i} \dot{x}^{k}(\zeta), a_{k}^{i} \dot{x}^{k}\left(J_{x}^{1} \xi\right), a_{k m}^{i} b_{j}^{m} \dot{x}^{k}\left(J_{x}^{1} \xi\right)+a_{k}^{i} b_{j}^{m} \dot{x}_{m}^{k}\left(J_{x}^{1} \xi\right)\right) \\
=a_{k}^{l} \Xi_{(U, \varphi)}^{k}\left(\zeta, J_{x}^{1} \xi\right), \\
\quad \dot{\Xi}_{(V, \psi)}^{l}\left(a_{k}^{i} \dot{x}^{k}(\zeta), a_{k}^{i} \dot{x}^{k}\left(J_{x}^{1} \xi\right), a_{k m}^{i} b_{j}^{m} \dot{x}^{k}\left(J_{x}^{1} \xi\right)\right. \\
\left.\quad+a_{k}^{i} b_{j}^{m} \dot{x}_{m}^{k}\left(J_{x}^{1} \xi\right)\right) \\
=a_{j k}^{l} \dot{x}^{j}(\zeta) \Xi_{(U, \varphi)}^{k}\left(\zeta, J_{x}^{1} \xi\right)+a_{k}^{l} \dot{\Xi}_{(U, \varphi)}^{k}\left(\zeta, J_{x}^{1} \xi\right) .
\end{gathered}
$$

In the well-known sense, the canonical vector fields are completely determined by certain differential invariants, that is, equivariant mappings from the type fibre $\mathbf{R}^{n} \times \mathbf{R}^{n} \times \mathbf{R}^{n^{2}}$ of $\tau_{X}^{*} J^{1} T X$ into the type fibre $\mathbf{R}^{n} \times \mathbf{R}^{n} \times \mathbf{R}^{n}$ of $T^{2} X$ over $X$ with respect to the canonical actions of the differential group $L_{n}^{2}$, induced by diffeomorphisms of $X$. We can characterize these actions explicitly in terms of the first and second canonical coordinates $a_{j}^{i}, a_{j k}^{i}$ and $b_{r}^{p}, b_{r s}^{p}$ on the differential group $L_{n}^{2}$. Note that

$$
a_{p}^{l} b_{r}^{p}=\delta_{r}^{l}, \quad a_{p q}^{l} b_{r}^{p}+a_{p}^{l} a_{q}^{s} b_{r s}^{p}=0 .
$$

If $A=\left(a_{j}^{i}, a_{j k}^{i}\right)$ is an element of the differential group $L_{n}^{2}$, denote by $\alpha_{A}$ (resp., $\beta_{A}$ ) the transformation of $\mathbf{R}^{n} \times \mathbf{R}^{n} \times \mathbf{R}^{n^{2}}$ (resp., $\mathbf{R}^{n} \times \mathbf{R}^{n} \times \mathbf{R}^{n}$ ), defined by $A$. For any points $\left(\zeta^{i}, \xi^{i}, \xi_{j}^{i}\right) \in$ $\mathbf{R}^{n} \times \mathbf{R}^{n} \times \mathbf{R}^{n^{2}}$ and $\left(Z^{i}, \Xi^{i}, \dot{\Xi}^{i}\right) \in \mathbf{R}^{n} \times \mathbf{R}^{n} \times \mathbf{R}^{n}$, we denote

$$
\begin{gathered}
\left(\bar{\zeta}^{i}, \bar{\xi}^{i}, \bar{\xi}_{j}^{i}\right)=\alpha_{A}\left(\zeta^{i}, \xi^{i}, \xi_{j}^{i}\right), \\
\left(\bar{Z}^{i}, \bar{\Xi}^{i}, \overline{\dot{\Xi}}^{i}\right)=\beta_{A}\left(Z^{i}, \Xi^{i}, \dot{\Xi}^{i}\right) .
\end{gathered}
$$

The following lemma defines the points (24) explicitly.

Lemma 4. Let $A=\left(a_{j}^{i}, a_{j k}^{i}\right)$ be an element of the differential group $L_{n}^{2}$.

(a) The canonical group action of $L_{n}^{2}$ on $\mathbf{R}^{n} \times \mathbf{R}^{n} \times \mathbf{R}^{n^{2}}$ is given by the equations:

$$
\begin{aligned}
& \bar{\zeta}^{i}=a_{k}^{i} \zeta^{k}, \quad \bar{\xi}^{i}=a_{k}^{i} \xi^{k}, \\
& \bar{\xi}_{j}^{i}=a_{k m}^{i} b_{j}^{m} \xi^{k}+a_{k}^{i} b_{j}^{m} \xi_{m}^{k} .
\end{aligned}
$$

(b) The canonical group action of $L_{n}^{2}$ on $\mathbf{R}^{n} \times \mathbf{R}^{n} \times \mathbf{R}^{n}$ is given by the equations:

$$
\begin{gathered}
\bar{Z}^{i}=a_{k}^{i} Z^{k}, \quad \bar{\Xi}^{i}=a_{k}^{i} \Xi^{k}, \\
\dot{\Xi}^{i}=a_{j k}^{i} \zeta^{j} \Xi^{k}+a_{k}^{i} \dot{\Xi}^{k} .
\end{gathered}
$$

Proof. The left $L_{n}^{2}$-actions on the type fibres of $\tau_{X}^{*} J^{1} T X$ and $T^{2} X$ can be derived from the general theory of differential groups and differential invariants [13]. Note that these $L_{n}^{2}$ actions can also be defined by transformation properties of components of jets and tangent vectors; compare with Lemmas 1 and 2 with the substitution

$$
\begin{array}{ll}
a_{k}^{l}=\frac{\partial y^{l}}{\partial x^{k}}, & a_{k p}^{l}=\frac{\partial^{2} y^{l}}{\partial x^{p} \partial x^{k}}, \\
b_{r}^{p}=\frac{\partial x^{p}}{\partial y^{r}}, & b_{r s}^{p}=\frac{\partial^{2} x^{p}}{\partial y^{r} \partial y^{s}} .
\end{array}
$$

Since a canonical vector field is always a morphism over the identity mapping $\mathrm{id}_{T X}$, the corresponding differential invariant $F: \mathbf{R}^{n} \times \mathbf{R}^{n} \times \mathbf{R}^{n^{2}} \rightarrow \mathbf{R}^{n} \times \mathbf{R}^{n} \times \mathbf{R}^{n}$ has an expression $\left(\zeta^{i}, \xi^{i}, \xi_{j}^{i}\right) \rightarrow F\left(\zeta^{i}, \xi^{i}, \xi_{j}^{i}\right)=\left(\zeta^{i}, F^{j}\left(\zeta^{i}, \xi^{i}, \xi_{j}^{i}\right), \dot{F}^{j}\left(\zeta^{i}, \xi^{i}, \xi_{j}^{i}\right)\right)$; we denote $F=\left(F^{i}, \dot{F}^{i}\right)$.

Theorem 5. A mapping $F=\left(F^{i}, \dot{F}^{i}\right)$ is a differential invariant if and only if

$$
\begin{aligned}
& F^{i}\left(a_{s}^{p} \zeta^{s}, a_{s}^{p} \xi^{s}, a_{s m}^{p} b_{q}^{s} \xi^{m}+a_{m}^{p} b_{q}^{s} \xi_{s}^{m}\right) \\
& =a_{k}^{i} F^{k}\left(\zeta^{s}, \xi^{s}, \xi_{s}^{m}\right), \\
& \dot{F}^{i}\left(a_{s}^{p} \zeta^{s}, a_{s}^{p} \xi^{s}, a_{s m}^{p} b_{q}^{s} \xi^{m}+a_{m}^{p} b_{q}^{s} \xi_{s}^{m}\right) \\
& =a_{k p}^{i} \zeta^{p} F^{k}\left(\zeta^{s}, \xi^{s}, \xi_{s}^{m}\right)+a_{k}^{i} \dot{F}^{k}\left(\zeta^{s}, \xi^{s}, \xi_{s}^{m}\right),
\end{aligned}
$$

for all $\left(\zeta^{s}, \xi^{s}, \xi_{s}^{m}\right) \in \mathbf{R}^{n} \times \mathbf{R}^{n} \times \mathbf{R}^{n^{2}}$ and $\left(a_{j}^{i}, a_{j k}^{i}\right) \in L_{n}^{2}$.

Proof. Equations (28) are direct consequences of (25) and (26).

Remark 6. Note that our definition of the canonical vector field differs from the lifting of a vector field from a base manifold to its tangent bundle, which is defined by means of the lifting of diffeomorphisms and 1-parameter groups of diffeomorphisms to the tangent bundle $[2,9]$.

Remark 7. We can specify Theorem 3 to diffeomorphisms $\alpha$, preserving a given point $x \in X$, such that $\alpha(x)=x$, and to charts $(U, \varphi)$ and $(V, \psi)$ such that $(U, \varphi)=(V, \psi)$ and $\varphi(x)=0$. Then, the components $\Xi_{(U, \varphi)}^{l}$ and $\dot{\Xi}_{(U, \varphi)}^{k}$ of a canonical vector field $\Xi$ satisfy

$$
\begin{aligned}
& \Xi_{(U, \varphi)}^{l}\left(a_{k}^{i} \zeta^{k}, a_{k}^{i} \xi^{k}, a_{k m}^{i} b_{j}^{m} \xi^{k}+a_{k}^{i} b_{j}^{m} \xi_{m}^{k}\right) \\
& =a_{k}^{l} \Xi_{(U, \varphi)}^{k}\left(\zeta^{k}, \xi^{k}, \xi_{m}^{k}\right), \\
& \dot{\Xi}_{(U, \varphi)}^{l}\left(a_{k}^{i} \zeta^{k}, a_{k}^{i} \xi^{k}, a_{k m}^{i} b_{j}^{m} \xi^{k}+a_{k}^{i} b_{j}^{m} \xi_{m}^{k}\right) \\
& \quad=a_{j k}^{l} \zeta^{j} \Xi_{(U, \varphi)}^{k}\left(\zeta^{k}, \xi^{k}, \xi_{m}^{k}\right)+a_{k}^{l} \dot{\Xi}_{(U, \varphi)}^{k}\left(\zeta^{k}, \xi^{k}, \xi_{m}^{k}\right),
\end{aligned}
$$


where

$$
\begin{gathered}
a_{k}^{l}=\left(\frac{\partial\left(x^{l} \alpha \varphi^{-1}\right)}{\partial x^{k}}\right)_{0} \quad a_{j k}^{l}=\left(\frac{\partial^{2}\left(x^{l} \alpha \varphi^{-1}\right)}{\partial x^{j} \partial x^{k}}\right)_{0}, \\
b_{j}^{k}=\left(\frac{\partial\left(x^{k} \alpha^{-1} \psi^{-1}\right)}{\partial x^{j}}\right)_{0} .
\end{gathered}
$$

\section{Canonical Vector Fields: Classification}

We find all solutions $F=\left(F^{i}, \dot{F}^{i}\right)$ of the equations for differential invariants, associated with canonical vector fields $\Xi: \tau_{X}^{*} J^{1} T X \rightarrow T^{2} X$ (Theorem 5). These equations can be written in coordinates as

$$
\begin{aligned}
& F^{i}\left(a_{s}^{p} \dot{x}^{s}, a_{s}^{p} \dot{z}^{s}, a_{s m}^{p} b_{q}^{s} \dot{z}^{m}+a_{m}^{p} b_{q}^{s} \dot{z}_{s}^{m}\right) \\
& \quad=a_{k}^{i} F^{k}\left(\dot{x}^{s}, \dot{z}^{s}, \dot{z}_{s}^{m}\right), \\
& \dot{F}^{i}\left(a_{s}^{p} \dot{x}^{s}, a_{s}^{p} \dot{z}^{s}, a_{s m}^{p} b_{q}^{s} \dot{z}^{m}+a_{m}^{p} b_{q}^{s} \dot{z}_{s}^{m}\right) \\
& \quad=a_{k p}^{i} \dot{x}^{p} F^{k}\left(\dot{x}^{s}, \dot{z}^{s}, \dot{z}_{s}^{m}\right)+a_{k}^{i} \dot{F}^{k}\left(\dot{x}^{s}, \dot{z}^{s}, \dot{z}_{s}^{m}\right) .
\end{aligned}
$$

The following lemma solves (31).

Lemma 8. The functions $F^{i}$ satisfy condition (31) if and only if

$$
F^{i}\left(\dot{x}^{j}, \dot{z}^{j}, \dot{z}_{k}^{j}\right)=A \dot{x}^{i}+B \dot{z}^{i},
$$

for some constants $A, B \in \mathbf{R}$.

Proof. (1) First we consider (31) for the group elements, belonging to the subgroup $K_{n}^{2}$ of $L_{n}^{2}$; equations of $K_{n}^{2}$ are

$$
a_{s}^{p}=\delta_{s}^{p},
$$

and we get the system

$$
F^{i}\left(\dot{x}^{p}, \dot{z}^{p}, a_{q m}^{p} \dot{z}^{m}+\dot{z}_{q}^{p}\right)=F^{i}\left(\dot{x}^{s}, \dot{z}^{s}, \dot{z}_{s}^{m}\right) .
$$

This equation is obviously satisfied at all points where $\dot{z}^{m}=0$. On the other hand, suppose that there exists at least one index $j$ such that $\dot{z}^{j} \neq 0$; then to every point $\dot{y}_{q}^{p}$ one can find the group parameters $a_{q m}^{p}$ such that $a_{q m}^{p} \dot{z}^{m}+\dot{z}_{q}^{p}=\dot{y}_{q}^{p}$. Indeed, if, for example, $\dot{z}^{1} \neq 0$, we set

$$
a_{q, 1}^{p}=\frac{\dot{y}_{q}^{p}-\dot{z}_{q}^{p}}{\dot{z}^{1}}, \quad a_{q, 2}^{p}, a_{q, 3}^{p}, \ldots, a_{q, n}^{p}=0 .
$$

This choice of the group parameters yields

$$
F^{i}\left(\dot{x}^{p}, \dot{z}^{p}, \dot{y}_{q}^{p}\right)=F^{i}\left(\dot{x}^{p}, \dot{z}^{p}, \dot{z}_{s}^{m}\right),
$$

which shows that $F^{i}\left(\dot{x}^{j}, \dot{x}^{j}, \dot{x}_{k}^{j}\right)$ is independent of $\dot{x}_{q}^{p}$.

(2) In view of (37), we can write $F^{i}\left(\dot{x}^{j}, \dot{x}^{j}\right)$ instead of $F^{i}\left(\dot{x}^{j}, \dot{x}^{j}, \dot{x}_{k}^{j}\right)$. Turning back to conditions (31), we have the following equations for the functions $F^{i}$ :

$$
F^{i}\left(a_{s}^{j} \dot{x}^{s}, a_{s}^{j} \dot{z}^{s}\right)=a_{k}^{i} F^{k}\left(\dot{x}^{j}, \dot{z}^{j}\right) .
$$

Properties of $F^{i}$ are completely determined by the subgroup $L_{n}^{1}$ of $L_{n}^{2}$. If $a_{s}^{p}=\lambda \delta_{s}^{p}, \lambda \neq 0$, then by (38) $F^{i}$ satisfies the positive homogeneity condition

$$
F^{i}\left(\lambda \dot{x}^{j}, \lambda \dot{z}^{k}\right)=\lambda F^{i}\left(\dot{x}^{j}, \dot{z}^{k}\right) .
$$

We suppose, however, that the functions $F^{i}$ are defined at the origin $\dot{x}^{p}=0, \dot{z}^{p}=0$; then it is easily seen that (39) also holds for $\lambda=0$. Indeed, in this case, we have $F^{i}(\lambda \cdot 0, \lambda \cdot 0)=$ $F^{i}(0,0)=\lambda F^{i}(0,0)$ for all $\lambda \neq 0$, hence $F^{i}(0,0)=0$. On the other hand, the points $\lambda \dot{x}^{j}$ and $\lambda \dot{z}^{k}$ are always defined for $\lambda=$ 0 ; then $F^{i}\left(0 \cdot \dot{x}^{j}, 0 \cdot \dot{z}^{k}\right)=F^{i}(0,0)=0=0 \cdot F^{i}\left(\dot{x}^{j}, \dot{z}^{k}\right)$, which proves (39) for $\lambda=0$.

Then, we have from (28) $D_{1, q} F^{i}\left(\lambda \dot{x}^{l}, \lambda \dot{z}^{l}\right) \dot{x}^{q}+$ $D_{2, q} F^{i}\left(\lambda \dot{x}^{l}, \lambda \dot{z}^{l}\right) \dot{z}^{q}=F^{i}\left(\dot{x}^{l}, \dot{z}^{l}\right)$ by differentiation with respect to $\lambda$; we see that the expression on the left does not depend on $\lambda$. For $\lambda=0$, we get $D_{1, q} F^{i}(0,0) \dot{x}^{q}+D_{2, q} F^{i}(0,0) \dot{z}^{q}=F^{i}\left(\dot{x}^{l}, \dot{z}^{l}\right)$, showing that $F^{i}$ is linear in $\dot{x}^{q}$ and $\dot{z}^{q}$; that is,

$$
F^{i}\left(\dot{x}^{j}, \dot{z}^{j}\right)=S_{q}^{i} \dot{x}^{q}+T_{q}^{i} \dot{z}^{i}
$$

Substituting now into (38), we get

$$
S_{q}^{i} a_{s}^{q} \dot{x}^{s}+T_{q}^{i} a_{s}^{q} \dot{z}^{s}=a_{k}^{i}\left(S_{q}^{k} \dot{x}^{q}+T_{q}^{k} \dot{z}^{q}\right) .
$$

That is, $S_{q}^{i} a_{s}^{q}=a_{q}^{i} S_{s}^{q}$ and $T_{q}^{i} a_{s}^{q}=a_{q}^{i} T_{s}^{q}$. Thus, $S_{q}^{i} a_{l}^{q} \delta_{s}^{l}=\delta_{l}^{i} a_{q}^{l} S_{s}^{q}$, hence $S_{q}^{i} \delta_{s}^{l}=\delta_{q}^{i} S_{s}^{l}$ and by the trace operation, $n S_{q}^{i}=\delta_{q}^{i} S_{l}^{l}$. Analogously, $n T_{q}^{i}=\delta_{q}^{i} T_{l}^{l}$. These expressions together with (40) prove formula (33).

(3) If condition (33) is satisfied, then we get (31) by immediate substitution.

Now, we wish to solve (32). In view of Lemma 8, these equations are of the form

$$
\begin{aligned}
& \dot{F}^{i}\left(a_{s}^{p} \dot{x}^{s}, a_{s}^{p} \dot{z}^{s}, a_{s m}^{p} b_{q}^{s} \dot{z}^{m}+a_{m}^{p} b_{q}^{s} \dot{z}_{s}^{m}\right) \\
& \quad=a_{k p}^{i} \dot{x}^{p}\left(A \dot{x}^{k}+B \dot{z}^{k}\right)+a_{k}^{i} \dot{F}^{k}\left(\dot{x}^{s}, \dot{z}^{s}, \dot{z}_{s}^{m}\right) .
\end{aligned}
$$

We prove separately the following lemma.

Lemma 9. The following two conditions are equivalent:

(a) The functions $Q_{p}^{i q}=Q_{p}^{i q}\left(\dot{x}^{j}, \dot{z}^{j}\right)$ satisfy

$$
Q_{p}^{i l}\left(a_{s}^{j} \dot{x}^{s}, a_{s}^{j} \dot{z}^{s}\right) a_{m}^{p}=a_{k}^{l} a_{s}^{i} Q_{m}^{s k}\left(\dot{x}^{j}, \dot{z}^{j}\right) .
$$

(b) The functions $Q_{p}^{i q}$ are of the form

$$
\begin{aligned}
Q_{m}^{i k}\left(\dot{x}^{j}, \dot{z}^{j}\right) & \\
= & \left(P\left(\delta_{j}^{k} \delta_{m}^{i}+\delta_{m}^{k} \delta_{j}^{i}\right)+Q\left(\delta_{j}^{k} \delta_{m}^{i}-\delta_{m}^{k} \delta_{j}^{i}\right)\right) \dot{x}^{j} \\
& +\left(R\left(\delta_{j}^{k} \delta_{m}^{i}+\delta_{m}^{k} \delta_{j}^{i}\right)+S\left(\delta_{j}^{k} \delta_{m}^{i}-\delta_{m}^{k} \delta_{j}^{i}\right)\right) \dot{z}^{j},
\end{aligned}
$$

where $P, Q, R, S \in \mathbf{R}$. 
Proof. (1) If $a_{j}^{i}=\lambda \delta_{j}^{i}$, Lemma 9, equation (43) $\lambda Q_{m}^{i l}\left(\lambda \dot{x}^{j}, \lambda \dot{z}^{j}\right)$ $=\lambda^{2} Q_{m}^{i l}\left(\dot{x}^{j}, \dot{z}^{j}\right)$ yields

$$
Q_{m}^{i l}\left(\dot{x}^{j}, \dot{z}^{j}\right)=S_{m j}^{i l} \dot{x}^{j}+T_{m j}^{i l} \dot{z}^{j},
$$

for some $S_{m j}^{i l}$, $S_{m j}^{i l} \in \mathbf{R}$ (Lemma 8). Substituting back to (43), we get conditions for the coefficients $S_{m j}^{i l}, S_{m j}^{i l} \in \mathbf{R}$ :

$$
\left(S_{s j}^{i l} a_{k}^{j} \dot{x}^{k}+T_{s j}^{i l} a_{k}^{j} \dot{z}^{k}\right) a_{m}^{s}=a_{t}^{l} a_{k}^{i}\left(S_{m j}^{k t} \dot{x}^{j}+T_{m j}^{t k} \dot{z}^{j}\right),
$$

or, which is the same, $\left(S_{s j}^{i l} \dot{x}^{t}+T_{s j}^{i l} \dot{z}^{t}\right) \delta_{m}^{k} a_{t}^{j} a_{k}^{s}=\delta_{j}^{j} \delta_{s}^{i}\left(S_{m r}^{k t} \dot{x}^{r}+\right.$ $\left.T_{m r}^{t k} \dot{z}^{r}\right) a_{t}^{j} a_{k}^{s}$; that is,

$$
S_{p j}^{i l} a_{t}^{j} a_{m}^{p}=a_{k}^{l} a_{s}^{i} S_{m t}^{s k}, \quad T_{p j}^{i l} a_{t}^{j} a_{m}^{p}=a_{k}^{l} a_{s}^{i} T_{m t}^{s k} .
$$

To determine the constants $S_{p j}^{i l}$, we write $S_{p j}^{i l} \delta_{t}^{s} \delta_{m}^{k} a_{s}^{j} a_{k}^{p}=$ $a_{k}^{j} a_{s}^{p} \delta_{j}^{l} \delta_{p}^{i} S_{m t}^{s k}$ from which it follows that

$$
S_{p j}^{i l} \delta_{t}^{s} \delta_{m}^{k}+S_{j p}^{i l} \delta_{t}^{k} \delta_{m}^{s}=\delta_{j}^{l} \delta_{p}^{i} S_{m t}^{s k}+\delta_{p}^{l} \delta_{j}^{i} S_{m t}^{k s} .
$$

We apply to this formula various trace operations. Contractions in $s, t$ and then in $k, m$ yield $n S_{p j}^{i l} \delta_{m}^{k}+S_{j p}^{i l} \delta_{m}^{k}=\delta_{j}^{l} \delta_{p}^{i} S_{m s}^{s k}+$ $\delta_{p}^{l} \delta_{j}^{i} S_{m s}^{k s}$ and

$$
n^{2} S_{p j}^{i l}+n S_{j p}^{i l}=\delta_{j}^{l} \delta_{p}^{i} S_{m s}^{s m}+\delta_{p}^{l} \delta_{j}^{i} S_{m s}^{m s}
$$

Contractions in $s, m$ and in $k, t$ yield $S_{p j}^{i l} \delta_{t}^{k}+n S_{j p}^{i l} \delta_{t}^{k}=$ $\delta_{j}^{l} \delta_{p}^{i} S_{m t}^{m k}+\delta_{p}^{l} \delta_{j}^{i} S_{m t}^{k m}$ and $n S_{p j}^{i l}+n^{2} S_{j p}^{i l}=\delta_{j}^{l} \delta_{p}^{i} S_{m t}^{m t}+\delta_{p}^{l} \delta_{j}^{i} S_{m t}^{t m}$. From these formulas, we find

$$
\begin{aligned}
& n(n+1)\left(S_{p j}^{i l}+S_{j p}^{i l}\right)=\left(\delta_{j}^{l} \delta_{p}^{i}+\delta_{p}^{l} \delta_{j}^{i}\right)\left(S_{m s}^{m s}+S_{m t}^{t m}\right), \\
& n(n-1)\left(S_{p j}^{i l}-S_{j p}^{i l}\right)=\left(\delta_{j}^{l} \delta_{p}^{i}-\delta_{p}^{l} \delta_{j}^{i}\right)\left(S_{m t}^{t m}+S_{m t}^{m t}\right), \\
& S_{p j}^{i l}+S_{j p}^{i l}=\frac{1}{n(n+1)}\left(\delta_{j}^{l} \delta_{p}^{i}+\delta_{p}^{l} \delta_{j}^{i}\right)\left(S_{m s}^{m s}+S_{m t}^{t m}\right), \\
& S_{p j}^{i l}-S_{j p}^{i l}=\frac{1}{n(n-1)}\left(\delta_{j}^{l} \delta_{p}^{i}-\delta_{p}^{l} \delta_{j}^{i}\right)\left(S_{m t}^{t m}+S_{m t}^{m t}\right),
\end{aligned}
$$

hence

$$
\begin{aligned}
S_{p j}^{i l}= & \frac{1}{2 n(n+1)}\left(\delta_{j}^{l} \delta_{p}^{i}+\delta_{p}^{l} \delta_{j}^{i}\right)\left(S_{m s}^{m s}+S_{m t}^{t m}\right), \\
& +\frac{1}{2 n(n-1)}\left(\delta_{j}^{l} \delta_{p}^{i}-\delta_{p}^{l} \delta_{j}^{i}\right)\left(S_{m t}^{t m}+S_{m t}^{m t}\right) .
\end{aligned}
$$

The same computation applies to $T_{p j}^{i l}$. Thus,

$$
\begin{aligned}
& S_{p j}^{i l}=P\left(\delta_{j}^{l} \delta_{p}^{i}+\delta_{p}^{l} \delta_{j}^{i}\right)+Q\left(\delta_{j}^{l} \delta_{p}^{i}-\delta_{p}^{l} \delta_{j}^{i}\right), \\
& T_{p j}^{i l}=R\left(\delta_{j}^{l} \delta_{p}^{i}+\delta_{p}^{l} \delta_{j}^{i}\right)+S\left(\delta_{j}^{l} \delta_{p}^{i}-\delta_{p}^{l} \delta_{j}^{i}\right),
\end{aligned}
$$

for some constants $P, Q, R, S \in \mathbf{R}$. Formula (44) now follows from (52) and (45).
(2) Conversely, suppose that $Q_{m}^{i k}$ satisfies (44). Writing

$$
\begin{aligned}
Q_{p}^{i q}=( & \left.P\left(\delta_{j}^{q} \delta_{p}^{i}+\delta_{p}^{q} \delta_{j}^{i}\right)+Q\left(\delta_{j}^{q} \delta_{p}^{i}-\delta_{p}^{q} \delta_{j}^{i}\right)\right) \dot{x}^{j} \\
& +\left(R\left(\delta_{j}^{q} \delta_{p}^{i}+\delta_{p}^{q} \delta_{j}^{i}\right)+S\left(\delta_{j}^{q} \delta_{p}^{i}-\delta_{p}^{q} \delta_{j}^{i}\right)\right) \dot{z}^{j}, \\
Q_{m}^{s k}=( & \left.P\left(\delta_{j}^{k} \delta_{m}^{s}+\delta_{m}^{k} \delta_{j}^{s}\right)+Q\left(\delta_{j}^{k} \delta_{m}^{s}-\delta_{m}^{k} \delta_{j}^{s}\right)\right) \dot{x}^{j} \\
& +\left(R\left(\delta_{j}^{k} \delta_{m}^{s}+\delta_{m}^{k} \delta_{j}^{s}\right)+S\left(\delta_{j}^{k} \delta_{m}^{s}-\delta_{m}^{k} \delta_{j}^{s}\right)\right) \dot{z}^{j}
\end{aligned}
$$

and substituting these expressions into (44), we have

$$
\begin{aligned}
Q_{p}^{i q}\left(a_{s}^{j} \dot{x}^{s}, a_{s}^{j} \dot{z}^{s}\right) a_{m}^{p} b_{q}^{k} & \\
= & P a_{m}^{i} \dot{x}^{k}+P \delta_{m}^{k} a_{t}^{i} \dot{x}^{t}+Q a_{m}^{i} \dot{x}^{k}-Q \delta_{m}^{k} a_{t}^{i} \dot{x}^{t} \\
& +R a_{m}^{i} \dot{z}^{k}+R \delta_{m}^{k} a_{t}^{i} \dot{z}^{t}+S a_{m}^{i} \dot{z}^{k}-S \delta_{m}^{k} a_{t}^{i} \dot{z}^{t}, \\
a_{s}^{i} Q_{m}^{s k} & \left.\dot{x}^{j}, \dot{z}^{j}\right) \\
= & P a_{m}^{i} \dot{x}^{k}+P \delta_{m}^{k} a_{s}^{i} \dot{x}^{s}+Q a_{m}^{i} \dot{x}^{k}-Q \delta_{m}^{k} a_{s}^{i} \dot{x}^{s} \\
& +R a_{m}^{i} \dot{z}^{k}+R \delta_{m}^{k} a_{s}^{i} \dot{z}^{s}+S a_{m}^{i} \dot{z}^{k}-S \delta_{m}^{k} a_{s}^{i} \dot{z}^{s},
\end{aligned}
$$

proving (13).

Now, we are in position to give a solution to (42).

Lemma 10. The functions $\dot{F}^{i}$ satisfy condition (42) if and only if

$$
\begin{gathered}
A=0, \\
\dot{F}^{i}\left(\dot{x}^{j}, \dot{z}^{j}, \dot{z}_{k}^{j}\right)=K \dot{x}^{i}+L \dot{z}^{i}+B \dot{x}^{r} \dot{z}_{r}^{i}
\end{gathered}
$$

for some constants $K, L \in \mathbf{R}$.

Proof. (1) Suppose that $\dot{F}^{i}$ satisfy (42). Then if $a_{s}^{p}=\delta_{s}^{p}$, we have

$$
\begin{aligned}
& \dot{F}^{i}\left(\dot{x}^{p}, \dot{z}^{p}, a_{q m}^{p} \dot{z}^{m}+\dot{z}_{q}^{p}\right) \\
& \quad=a_{k p}^{i} \dot{x}^{p}\left(A \dot{x}^{k}+B \dot{z}^{k}\right)+\dot{F}^{i}\left(\dot{x}^{s}, \dot{z}^{s}, \dot{z}_{s}^{m}\right) .
\end{aligned}
$$

Differentiating with respect to $\dot{z}_{s}^{r}$, we have

$$
\left(\frac{\partial \dot{F}^{i}}{\partial \dot{z}_{s}^{r}}\right)_{\left(\dot{x}^{p}, \dot{z}^{p}, a_{q m}^{p} \dot{z}^{m}+\dot{z}_{q}^{p}\right)}=\left(\frac{\partial \dot{F}^{i}}{\partial \dot{z}_{s}^{r}}\right)_{\left(\dot{x}^{p}, \dot{z}^{p}, \dot{z}_{q}^{p}\right)},
$$

which shows that the derivative $\partial \dot{F}^{i} / \partial \dot{z}_{s}^{r}$ does not depend on $\dot{z}_{q}^{p}$. Then, however,

$$
\dot{F}^{i}\left(\dot{x}^{j}, \dot{z}^{j}, \dot{z}_{k}^{j}\right)=P^{i}\left(\dot{x}^{j}, \dot{z}^{j}\right)+Q_{s}^{i r}\left(\dot{x}^{j}, \dot{z}^{j}\right) \dot{z}_{r}^{s},
$$

where the functions $P^{i}$ and $Q_{s}^{i r}$ do not depend on $\dot{z}_{q}^{p}$. Substituting from (59) back to (57), we have

$$
\begin{aligned}
& P^{i}\left(\dot{x}^{p}, \dot{z}^{p}\right)+Q_{s}^{i r}\left(\dot{x}^{p}, \dot{z}^{p}\right)\left(a_{r m}^{s} \dot{z}^{m}+\dot{z}_{r}^{s}\right) \\
& \quad=a_{k p}^{i} \dot{x}^{p}\left(A \dot{x}^{k}+B \dot{z}^{k}\right)+P^{i}\left(\dot{x}^{p}, \dot{z}^{p}\right)+Q_{s}^{i r}\left(\dot{x}^{p}, \dot{z}^{p}\right) \dot{z}_{r}^{s},
\end{aligned}
$$


hence $Q_{s}^{i r}\left(\dot{x}^{p}, \dot{z}^{p}\right) a_{r m}^{s} \dot{z}^{m}=\delta_{s}^{i} a_{r m}^{s} \dot{x}^{r}\left(A \dot{x}^{m}+B \dot{z}^{m}\right)$. Thus, the functions $P^{i}$ and $Q_{s}^{i r}$ in (59) must satisfy

$$
\begin{aligned}
& Q_{s}^{i r}\left(\dot{x}^{p}, \dot{z}^{p}\right) \dot{z}^{m}+Q_{s}^{i m}\left(\dot{x}^{p}, \dot{z}^{p}\right) \dot{z}^{r} \\
& \quad=\delta_{s}^{i} \dot{x}^{r}\left(A \dot{x}^{m}+B \dot{z}^{m}\right)+\delta_{s}^{i} \dot{x}^{m}\left(A \dot{x}^{r}+B \dot{z}^{r}\right) .
\end{aligned}
$$

Note that the trace operation in $i$ and $s$ yields

$$
\begin{aligned}
& \frac{1}{n}\left(Q_{s}^{s r}\left(\dot{x}^{p}, \dot{z}^{p}\right) \dot{z}^{m}+Q_{s}^{s m}\left(\dot{x}^{p}, \dot{z}^{p}\right) \dot{z}^{r}\right) \\
& \quad=\dot{x}^{r}\left(A \dot{x}^{m}+B \dot{z}^{m}\right)+\dot{x}^{m}\left(A \dot{x}^{r}+B \dot{z}^{r}\right) .
\end{aligned}
$$

(2) We now use (56) for the group parameters $a_{j k}^{i}=0$. We have the condition

$$
\dot{F}^{i}\left(a_{s}^{p} \dot{x}^{s}, a_{s}^{p} \dot{z}^{s}, a_{m}^{p} b_{q}^{s} \dot{z}_{s}^{m}\right)=a_{k}^{i} \dot{F}^{k}\left(\dot{x}^{s}, \dot{z}^{s}, \dot{z}_{s}^{m}\right),
$$

and, from (59),

$$
\begin{array}{r}
P^{i}\left(a_{s}^{j} \dot{x}^{s}, a_{s}^{j} \dot{z}^{s}\right)+Q_{s}^{i r}\left(a_{s}^{j} \dot{x}^{s}, a_{s}^{j} \dot{z}^{s}\right) a_{m}^{s} b_{r}^{t} \dot{z}_{t}^{m} \\
=a_{k}^{i} P^{k}\left(\dot{x}^{j}, \dot{z}^{j}\right)+a_{k}^{i} Q_{s}^{k r}\left(\dot{x}^{j}, \dot{z}^{j}\right) \dot{z}_{r}^{s} .
\end{array}
$$

These equations split to the system

$$
\begin{gathered}
P^{i}\left(a_{s}^{j} \dot{x}^{s}, a_{s}^{j} \dot{z}^{s}\right)=a_{k}^{i} P^{k}\left(\dot{x}^{j}, \dot{z}^{j}\right), \\
Q_{s}^{i r}\left(a_{s}^{j} \dot{x}^{s}, a_{s}^{j} \dot{z}^{s}\right) a_{m}^{s} b_{r}^{t}=a_{k}^{i} Q_{s}^{k r}\left(\dot{x}^{j}, \dot{z}^{j}\right) .
\end{gathered}
$$

Then, however,

$$
P^{i}\left(\dot{x}^{j}, \dot{z}^{j}\right)=K \dot{x}^{i}+L \dot{z}^{i},
$$

for some constants $K, L \in \mathbf{R}$ (Lemma 8), and

$$
\begin{aligned}
Q_{s}^{i r}\left(\dot{x}^{j}, \dot{z}^{j}\right) & \\
= & \left(P\left(\delta_{j}^{r} \delta_{s}^{i}+\delta_{s}^{r} \delta_{j}^{i}\right)+Q\left(\delta_{j}^{r} \delta_{s}^{i}-\delta_{s}^{r} \delta_{j}^{i}\right)\right) \dot{x}^{j} \\
& +\left(R\left(\delta_{j}^{r} \delta_{s}^{i}+\delta_{s}^{r} \delta_{j}^{i}\right)+S\left(\delta_{j}^{r} \delta_{s}^{i}-\delta_{s}^{r} \delta_{j}^{i}\right)\right) \dot{z}^{j},
\end{aligned}
$$

where $P, Q, R, S \in \mathbf{R}$ (Lemma 9). These functions satisfy (61); that is,

$$
\begin{gathered}
\left(P\left(\delta_{j}^{r} \delta_{s}^{i}+\delta_{s}^{r} \delta_{j}^{i}\right)+Q\left(\delta_{j}^{r} \delta_{s}^{i}-\delta_{s}^{r} \delta_{j}^{i}\right)\right) \dot{x}^{j} \dot{z}^{m} \\
=\delta_{s}^{i}\left(2 A \dot{x}^{m} \dot{x}^{r}+B\left(\dot{x}^{m} \dot{z}^{r}+\dot{x}^{r} \dot{z}^{m}\right)\right)
\end{gathered}
$$

The trace in $i$ and $s$ yields

$$
\begin{aligned}
(P(n+1) & +Q(n-1)) \dot{x}^{r} \dot{z}^{m}+(R(n+1)+S(n-1)) \dot{z}^{r} \dot{z}^{m} \\
& +(P(n+1)+Q(n-1)) \dot{x}^{m} \dot{z}^{r} \\
& +(R(n+1)+S(n-1)) \dot{z}^{m} \dot{z}^{r} \\
= & n\left(2 A \dot{x}^{m} \dot{x}^{r}+B\left(\dot{x}^{m} \dot{z}^{r}+\dot{x}^{r} \dot{z}^{m}\right)\right) .
\end{aligned}
$$

Consequently, $A=0$ and

$$
\begin{gathered}
(P(n+1)+Q(n-1))\left(\dot{x}^{r} \dot{z}^{m}+\dot{x}^{m} \dot{z}^{r}\right)=n B\left(\dot{x}^{m} \dot{z}^{r}+\dot{x}^{r} \dot{z}^{m}\right) \\
(R(n+1)+S(n-1)) \dot{z}^{m} \dot{z}^{r}=0,
\end{gathered}
$$

hence

$$
\begin{gathered}
A=0, \\
P(n+1)+Q(n-1)=n B, \\
R(n+1)+S(n-1)=0 .
\end{gathered}
$$

Then from (68),

$$
\begin{aligned}
\left(P\left(\delta_{j}^{r} \delta_{s}^{i}+\delta_{s}^{r} \delta_{j}^{i}\right)+Q\left(\delta_{j}^{r} \delta_{s}^{i}-\delta_{s}^{r} \delta_{j}^{i}\right)\right) \dot{x}^{j} \dot{z}^{m} \\
\quad+S\left(\frac{1-n}{n+1}\left(\delta_{j}^{r} \delta_{s}^{i}+\delta_{s}^{r} \delta_{j}^{i}\right)+\left(\delta_{j}^{r} \delta_{s}^{i}-\delta_{s}^{r} \delta_{j}^{i}\right)\right) \dot{z}^{j} \dot{z}^{m} \\
\quad+\left(P\left(\delta_{j}^{m} \delta_{s}^{i}+\delta_{s}^{m} \delta_{j}^{i}\right)+Q\left(\delta_{j}^{m} \delta_{s}^{i}-\delta_{s}^{m} \delta_{j}^{i}\right)\right) \dot{x}^{j} \dot{z}^{r} \\
\quad+S\left(\frac{1-n}{n+1}\left(\delta_{j}^{m} \delta_{s}^{i}+\delta_{s}^{m} \delta_{j}^{i}\right)+\left(\delta_{j}^{m} \delta_{s}^{i}-\delta_{s}^{m} \delta_{j}^{i}\right)\right) \dot{z}^{j} \dot{z}^{r} \\
=\delta_{s}^{i} B\left(\dot{x}^{m} \dot{z}^{r}+\dot{x}^{r} \dot{z}^{m}\right) .
\end{aligned}
$$

The terms containing $\dot{z}^{j} \dot{z}^{m}$ should vanish separately. Since these terms are

$$
\begin{gathered}
\frac{1}{n+1} S\left(-(n-1) \delta_{s}^{i} \dot{z}^{r} \dot{z}^{m}-(n-1) \delta_{s}^{r} \dot{z}^{i} \dot{z}^{m}\right. \\
\left.+(n+1) \delta_{s}^{i} \dot{z}^{r} \dot{z}^{m}-(n+1) \delta_{s}^{r} \dot{z}^{i} \dot{z}^{m}\right) \\
+\frac{1}{n+1} S\left(-(n-1) \delta_{s}^{i} \dot{z}^{m} \dot{z}^{r}-(n-1) \delta_{s}^{m} \dot{z}^{i} \dot{z}^{r}\right. \\
\left.+(n+1) \delta_{s}^{i} \dot{z}^{m} \dot{z}^{r}-(n+1) \delta_{s}^{m} \dot{z}^{i} \dot{z}^{r}\right) \\
=\frac{-2 n}{n+1} S\left(\delta_{s}^{r} \dot{z}^{i} \dot{z}^{m}+\delta_{s}^{m} \dot{z}^{i} \dot{z}^{r}\right),
\end{gathered}
$$

we have, from (71) and (73),

$$
S=0, \quad R=0 .
$$

Analogously, the terms with $\dot{x}^{j} \dot{z}^{m}$ should vanish separately; that is,

$$
\begin{aligned}
\left(P\left(\delta_{j}^{r} \delta_{s}^{i}+\delta_{s}^{r} \delta_{j}^{i}\right)+Q\left(\delta_{j}^{r} \delta_{s}^{i}-\delta_{s}^{r} \delta_{j}^{i}\right)\right) \dot{x}^{j} \dot{z}^{m} & \\
& +\left(P\left(\delta_{j}^{m} \delta_{s}^{i}+\delta_{s}^{m} \delta_{j}^{i}\right)+Q\left(\delta_{j}^{m} \delta_{s}^{i}-\delta_{s}^{m} \delta_{j}^{i}\right)\right) \dot{x}^{j} \dot{z}^{r} \\
= & \delta_{s}^{i} B\left(\dot{x}^{m} \dot{z}^{r}+\dot{x}^{r} \dot{z}^{m}\right) .
\end{aligned}
$$

Since this equation can be written as

$$
\begin{aligned}
(P+Q) & \delta_{s}^{i}\left(\dot{x}^{r} \dot{z}^{m}+\dot{x}^{m} \dot{z}^{r}\right)+(P-Q)\left(\delta_{s}^{r} \dot{x}^{i} \dot{z}^{m}+\delta_{s}^{m} \dot{x}^{i} \dot{z}^{r}\right) \\
= & \delta_{s}^{i} B\left(\dot{x}^{m} \dot{z}^{r}+\dot{x}^{r} \dot{z}^{m}\right),
\end{aligned}
$$


we get $P+Q=B$ and $P-Q=0$; thus,

$$
P=Q=\frac{1}{2} B
$$

Summarizing, we see that condition (42) implies, from (59), (66), (67), (71), (74), and (77),

$$
A=0 \text {, }
$$

(48) and

$$
\dot{F}^{i}\left(\dot{x}^{j}, \dot{z}^{j}, \dot{z}_{k}^{j}\right)=K \dot{x}^{i}+L \dot{z}^{i}+B \dot{x}^{r} \dot{z}_{r}^{i}
$$

(3) It remains to prove that conditions (55) and (56) imply (42). The left-hand side of (42) is

$$
\begin{aligned}
& \dot{F}^{i}\left(a_{s}^{p} \dot{x}^{s}, a_{s}^{p} \dot{z}^{s}, a_{s m}^{p} b_{q}^{s} \dot{z}^{m}+a_{m}^{p} b_{q}^{s} \dot{z}_{s}^{m}\right) \\
& \quad=K a_{s}^{i} \dot{x}^{s}+L a_{s}^{i} \dot{z}^{s}+B \dot{x}^{t}\left(a_{t m}^{i} \dot{z}^{m}+a_{m}^{i} \dot{z}_{t}^{m}\right),
\end{aligned}
$$

and the right-hand side is

$$
\begin{aligned}
& a_{k p}^{i} \dot{x}^{p}\left(A \dot{x}^{k}+B \dot{z}^{k}\right)+a_{k}^{i} \dot{F}^{k}\left(\dot{x}^{s}, \dot{z}^{s}, \dot{z}_{s}^{m}\right) \\
& \quad=a_{k}^{i} K \dot{x}^{k}+a_{k}^{i} L \dot{z}^{k}+B \dot{x}^{p}\left(a_{k p}^{i} \dot{z}^{k}+a_{k}^{i} \dot{z}_{p}^{k}\right) .
\end{aligned}
$$

These formulas already verify condition (42). The proof is complete.

We can now summarize our results in the following theorem.

Theorem 11. Let $X$ be a manifold and let $\Xi: \tau_{X}^{*} J^{1} T X \rightarrow T^{2} X$ be a morphism over $\mathrm{id}_{T X}$. The following two conditions are equivalent:

(a) $\Xi$ is a canonical vector field.

(b) For any chart $(U, \varphi), \varphi=\left(x^{i}\right)$, on $X$

$$
\Xi=B\left(\dot{z}^{i} \frac{\partial}{\partial x^{i}}+\dot{x}^{r} \dot{z}_{r}^{i} \frac{\partial}{\partial \dot{x}^{i}}\right)+\left(K \dot{x}^{i}+L \dot{z}^{i}\right) \frac{\partial}{\partial \dot{x}^{i}},
$$

where $B, K, L \in \mathbf{R}$ are arbitrary constants.

Proof. (1) We show that (a) implies (b). Suppose that we have a canonical vector field $\Xi$. Then, in any chart on $X$ the 2ndorder vector field $\Xi$ has an expression

$$
\begin{aligned}
& \Xi\left(\zeta, J_{x}^{1} \xi\right) \\
& \quad=\Xi_{(U, \varphi)}^{k}\left(\zeta, J_{x}^{1} \xi\right)\left(\frac{\partial}{\partial x^{k}}\right)_{\zeta}+\dot{\Xi}_{(U, \varphi)}^{k}\left(\zeta, J_{x}^{1} \xi\right)\left(\frac{\partial}{\partial \dot{x}^{k}}\right)_{\zeta}
\end{aligned}
$$

such that for any points $x, y \in X$, any charts $(U, \varphi), \varphi=\left(x^{i}\right)$, at $x$ and $(V, \psi), \psi=\left(y^{i}\right)$, at $y$, and any diffeomorphism $\alpha$ : $U \rightarrow V$ such that $\alpha(x)=y$,

$$
\begin{aligned}
& \Xi_{(V, \psi)}^{l}\left(a_{k}^{i} \zeta^{k}, a_{k}^{i} \xi^{k}, a_{k m}^{i} b_{j}^{m} \xi^{k}+a_{k}^{i} b_{j}^{m} \xi_{m}^{k}\right) \\
& \quad=a_{k}^{l} \Xi_{(U, \varphi)}^{k}\left(\zeta^{k}, \xi^{k}, \xi_{m}^{k}\right), \\
& \dot{\Xi}_{(V, \psi)}^{l}\left(a_{k}^{i} \zeta^{k}, a_{k}^{i} \xi^{k}, a_{k m}^{i} b_{j}^{m} \xi^{k}+a_{k}^{i} b_{j}^{m} \xi_{m}^{k}\right) \\
& \quad=a_{j k}^{l} \dot{x}^{j}(\zeta) \Xi_{(U, \varphi)}^{k}\left(\zeta^{k}, \xi^{k}, \xi_{m}^{k}\right)+a_{k}^{l} \dot{\Xi}_{(U, \varphi)}^{k}\left(\zeta^{k}, \xi^{k}, \xi_{m}^{k}\right) .
\end{aligned}
$$

(Theorem 3). If $x=y$ and $(U, \varphi)=(V, \psi)$, the components $\Xi_{(U, \varphi)}^{l}$ and $\dot{\Xi}_{(U, \varphi)}^{l}$ define a differential invariant $F=\left(F^{i}, \dot{F}^{i}\right)$ (Theorem 5); then, however, $\Xi$ must be of the form (82) (Lemmas 9 and 10).

(2) To prove that (b) implies (a), we first show that any two members of the family of vector fields (82) agree on intersection of their domains. Let $(U, \varphi), \varphi=\left(x^{i}\right)$, and $(V, \psi), \psi=\left(y^{i}\right)$, be two charts on $X$ such that $U \cap V \neq \emptyset$, let $\left(x^{i}, \dot{x}^{i}, \dot{z}^{i}, \dot{z}_{j}^{i}\right)$ and $\left(y^{i}, \dot{y}^{i}, \dot{u}^{i}, \dot{u}_{j}^{i}\right)$ be the corresponding coordinates on $\tau_{X}^{*} J^{1} T X$. We want to show that

$$
\begin{aligned}
& B\left(\dot{z}^{i} \frac{\partial}{\partial x^{i}}+\dot{x}^{r} \dot{z}_{r}^{i} \frac{\partial}{\partial \dot{x}^{i}}\right)+\left(K \dot{x}^{i}+L \dot{z}^{i}\right) \frac{\partial}{\partial \dot{x}^{i}} \\
& =B\left(\dot{u}^{i} \frac{\partial}{\partial y^{i}}+\dot{y}^{r} \dot{u}_{r}^{i} \frac{\partial}{\partial \dot{y}^{i}}\right)+\left(K \dot{y}^{i}+L \dot{u}^{i}\right) \frac{\partial}{\partial \dot{y}^{i}}
\end{aligned}
$$

that is, in the notation of Lemma 1 ,

$$
B \dot{u}^{l}=\frac{\partial y^{l}}{\partial x^{k}} B \dot{z}^{k},
$$

$$
\begin{aligned}
K \dot{y}^{l}+ & L \dot{u}^{l}+B \dot{y}^{r} \dot{u}_{r}^{l} \\
& =\frac{\partial^{2} y^{l}}{\partial x^{p} \partial x^{k}} B \dot{x}^{p} \dot{z}^{k}+\frac{\partial y^{l}}{\partial x^{k}}\left(K \dot{x}^{k}+L \dot{z}^{k}+\dot{x}^{r} \dot{z}_{r}^{k}\right) .
\end{aligned}
$$

Each element of the family of vector fields (82) defines a differential invariant $F=\left(F^{i}, \dot{F}^{i}\right)$, where

$$
F^{i}=B \dot{z}^{i}, \quad \dot{F}^{i}=K \dot{x}^{i}+L \dot{z}^{i}+B \dot{x}^{r} \dot{z}_{r}^{i} .
$$

Recall that

$$
\begin{aligned}
& F^{i}\left(a_{s}^{p} \dot{x}^{s}, a_{s}^{p} \dot{z}^{s}, a_{s m}^{p} b_{q}^{s} \dot{z}^{m}+a_{m}^{p} b_{q}^{s} \dot{z}_{s}^{m}\right) \\
& \quad=a_{k}^{i} F^{k}\left(\dot{x}^{s}, \dot{z}^{s}, \dot{z}_{s}^{m}\right), \\
& \dot{F}^{i}\left(a_{s}^{p} \dot{x}^{s}, a_{s}^{p} \dot{z}^{s}, a_{s m}^{p} b_{q}^{s} \dot{z}^{m}+a_{m}^{p} b_{q}^{s} \dot{z}_{s}^{m}\right) \\
& \quad=a_{k p}^{i} \zeta^{p} F^{k}\left(\dot{x}^{s}, \dot{z}^{s}, \dot{z}_{s}^{m}\right)+a_{k}^{i} \dot{F}^{k}\left(\dot{x}^{s}, \dot{z}^{s}, \dot{z}_{s}^{m}\right),
\end{aligned}
$$

(Theorem 5). Now the right-hand sides of (86) can be written as

$a_{k}^{l} F^{k}\left(\dot{x}^{i}, \dot{z}^{i}, \dot{z}_{r}^{i}\right), \quad a_{k p}^{l} F^{k}\left(\dot{x}^{i}, \dot{z}^{i}, \dot{z}_{r}^{i}\right) \dot{z}^{p}+a_{k}^{l} \dot{F}^{k}\left(\dot{x}^{i}, \dot{z}^{i}, \dot{z}_{r}^{i}\right)$, 
and the left-hand sides are

$$
\begin{aligned}
& F^{l}\left(\dot{y}^{i}, \dot{u}^{i}, \dot{u}_{r}^{i}\right) \\
& =F^{l}\left(a_{s}^{i} \dot{x}^{s}, a_{s}^{i} \dot{z}^{s},\left(a_{s m}^{i} \dot{x}^{m}+a_{m}^{i} \dot{x}_{s}^{m}\right) b_{r}^{s}\right), \\
& \dot{F}^{l}\left(\dot{y}^{i}, \dot{u}^{i}, \dot{u}_{r}^{i}\right) \\
& =\dot{F}^{l}\left(\dot{y}^{i}, \dot{u}^{i},\left(a_{s m}^{i} \dot{x}^{m}+a_{m}^{i} \dot{x}_{s}^{m}\right) b_{r}^{s}\right),
\end{aligned}
$$

where

$$
\begin{gathered}
a_{k}^{l}=\left(\frac{\partial y^{l}}{\partial x^{k}}\right)_{\Psi(\zeta)}, \\
a_{k p}^{l}=\left(\frac{\partial^{2} y^{l}}{\partial x^{p} \partial x^{k}}\right)_{\Psi(\zeta)}, \\
b_{r}^{p}=\left(\frac{\partial x^{p}}{\partial y^{r}}\right)_{\Phi(\zeta)} .
\end{gathered}
$$

Expressions (89) and (90) prove (86) as well as existence of $\Xi$.

(3) To complete the proof, it remains to show that the vector field $\Xi$ is a canonical vector field; to this purpose, we verify condition (2) of Theorem 3. Express the vectors $\Xi\left(\zeta, J_{x}^{1} \xi\right)$ and $\Xi\left(T_{x} \alpha \cdot \zeta, J_{\alpha(x)}^{1}\left(T \alpha \circ \xi \circ \alpha^{-1}\right)\right)$ as in formula (15), Section 4

$$
\begin{aligned}
& \Xi\left(\zeta, J_{x}^{1} \xi\right) \\
& =\Xi_{(U, \varphi)}^{k}\left(\zeta, J_{x}^{1} \xi\right)\left(\frac{\partial}{\partial x^{k}}\right)_{\zeta}+\dot{\Xi}_{(U, \varphi)}^{k}\left(\zeta, J_{x}^{1} \xi\right)\left(\frac{\partial}{\partial \dot{x}^{k}}\right)_{\zeta} \\
& \Xi\left(T_{x} \alpha \cdot \zeta, J_{\alpha(x)}^{1}\left(T \alpha \circ \xi \circ \alpha^{-1}\right)\right) \\
& =\Xi_{(V, \psi)}^{k}\left(T_{x} \alpha \cdot \zeta, J_{\alpha(x)}^{1}\left(T \alpha \circ \xi \circ \alpha^{-1}\right)\right)\left(\frac{\partial}{\partial y^{k}}\right)_{\zeta} \\
& \quad+\dot{\Xi}_{(V, \psi)}^{k}\left(T_{x} \alpha \cdot \zeta, J_{\alpha(x)}^{1}\left(T \alpha \circ \xi \circ \alpha^{-1}\right)\right)\left(\frac{\partial}{\partial \dot{y}^{k}}\right)_{\zeta}
\end{aligned}
$$

We have already proved that

$$
\begin{aligned}
& \Xi=B \dot{z}^{k} \frac{\partial}{\partial x^{k}}+\left(K \dot{x}^{k}+L \dot{z}^{k}+B \dot{x}^{r} \dot{z}_{r}^{k}\right) \frac{\partial}{\partial \dot{x}^{k}}, \\
& \Xi=B \dot{u}^{k} \frac{\partial}{\partial y^{k}}+\left(K \dot{y}^{k}+L \dot{u}^{k}+B \dot{y}^{r} \dot{u}_{r}^{k}\right) \frac{\partial}{\partial \dot{y}^{k}},
\end{aligned}
$$

on the corresponding coordinate neighbourhoods. Thus, the components of these vector fields are

$$
\begin{array}{ll}
\Xi_{(U, \varphi)}^{i}=B \dot{z}^{i}, & \dot{\Xi}_{(U, \varphi)}^{k}=K \dot{x}^{k}+L \dot{z}^{k}+B \dot{x}^{r} \dot{z}_{r}^{k}, \\
\Xi_{(V, \psi)}^{i}=\widetilde{B} \dot{u}^{i}, & \dot{\Xi}_{(V, \psi)}^{k}=\widetilde{K} \dot{y}^{k}+\widetilde{L} \dot{u}^{k}+\widetilde{B} \dot{y}^{r} \dot{u}_{r}^{k} .
\end{array}
$$

We substitute these expressions into formulas (17) of Theorem 3,

$$
\begin{aligned}
& \Xi_{(V, \psi)}^{l}\left(a_{k}^{i} \zeta^{k}, a_{k}^{i} \xi^{k}, a_{k m}^{i} b_{j}^{m} \xi^{k}+a_{k}^{i} b_{j}^{m} \xi_{m}^{k}\right) \\
& =a_{k}^{l} \Xi_{(U, \varphi)}^{k}\left(\zeta^{i}, \xi^{i}, \xi_{j}^{i}\right), \\
& \dot{\Xi}_{(V, \psi)}^{l}\left(a_{k}^{i} \zeta^{k}, a_{k}^{i} \xi^{k}, a_{k m}^{i} b_{j}^{m} \xi^{k}+a_{k}^{i} b_{j}^{m} \xi_{m}^{k}\right) \\
& =a_{j k}^{l} \zeta^{j} \Xi_{(U, \varphi)}^{k}\left(\zeta^{i}, \xi^{i}, \xi_{m}^{i}\right)+a_{k}^{l} \dot{\Xi}_{(U, \varphi)}^{k}\left(\zeta^{i}, \xi^{i}, \xi_{m}^{i}\right) .
\end{aligned}
$$

Then since

$$
\begin{gathered}
\Xi_{(V, \psi)}^{l}\left(a_{k}^{i} \zeta^{k}, a_{k}^{i} \xi^{k}, a_{k m}^{i} b_{j}^{m} \xi^{k}+a_{k}^{i} b_{j}^{m} \xi_{m}^{k}\right)=\widetilde{B} a_{k}^{l} \xi^{k}, \\
a_{k}^{l} \Xi_{(U, \varphi)}^{k}\left(\zeta^{i}, \xi^{i}, \xi_{j}^{i}\right)=a_{k}^{l} B \xi^{k}, \\
\dot{\Xi}_{(V, \psi)}^{l}\left(a_{k}^{i} \zeta^{k}, a_{k}^{i} \xi^{k}, a_{k m}^{i} b_{j}^{m} \xi^{k}+a_{k}^{i} b_{j}^{m} \xi_{m}^{k}\right) \\
=\widetilde{K} a_{l}^{k} \zeta^{l}+\widetilde{L} a_{l}^{k} \xi^{l}+\widetilde{B} a_{p}^{r} \zeta^{p}\left(a_{l m}^{k} b_{r}^{m} \xi^{l}+a_{l}^{k} b_{r}^{m} \xi_{m}^{l}\right), \\
a_{j k}^{l} \zeta^{j} \Xi_{(U, \varphi)}^{k}\left(\zeta^{i}, \xi^{i}, \xi_{m}^{i}\right)+a_{k}^{l} \dot{\Xi}_{(U, \varphi)}^{k}\left(\zeta^{i}, \xi^{i}, \xi_{m}^{i}\right) \\
=B a_{j l}^{k} \zeta^{j} \xi^{l}+a_{l}^{k}\left(K \zeta^{l}+L \xi^{l}+B \zeta^{r} \xi_{r}^{l}\right),
\end{gathered}
$$

we have

$$
\begin{gathered}
\widetilde{B} a_{k}^{l} \xi^{k}=B a_{k}^{l} \xi^{k}, \\
\widetilde{K} a_{l}^{k} \zeta^{l}+\widetilde{L} a_{l}^{k} \xi^{l}+\widetilde{B} a_{p}^{r} \zeta^{p}\left(a_{l m}^{k} b_{r}^{m} \xi^{l}+a_{l}^{k} b_{r}^{m} \xi_{m}^{l}\right) \\
=B a_{j l}^{k} \zeta^{j} \xi^{l}+a_{l}^{k}\left(K \zeta^{l}+L \xi^{l}+B \zeta^{r} \xi_{r}^{l}\right) .
\end{gathered}
$$

But these conditions are equivalent to $\widetilde{B}=B, \widetilde{K}=K$, and $\widetilde{L}=L$ as required.

Having in mind that the canonical constructions are geometric constructions independent of charts, we can also state our theorem in an equivalent way as follows. Let $X$ be an $n$-dimensional manifold. Then for any vector field $\zeta$ on $X$, there are exactly three independent canonical vector fields $Z$ on $T X$ that can canonically be constructed from $\zeta$. If in a chart

$$
\zeta=\zeta^{i} \frac{\partial}{\partial x^{i}}
$$

then

$$
Z=B\left(\zeta^{i} \frac{\partial}{\partial x^{i}}+\dot{x}^{r} \frac{\partial \zeta^{i}}{\partial x^{r}} \frac{\partial}{\partial \dot{x}^{i}}\right)+\left(K \dot{x}^{i}+L \zeta^{i}\right) \frac{\partial}{\partial \dot{x}^{i}},
$$

where $B, K, L \in \mathbf{R}$ are arbitrary constants. Taking $K, L=0$ and $B=1$, we get the variational vector field; if $B, L=0$ and $K=1$, we get the Liouville vector field, and, if $B, K=0$ and $L=1$, we have the vertical lift vector field.

\section{Acknowledgments}

The first author (T. Li) is grateful for the support of the National Natural Science Foundation of China (Grant no. 
10801006). The second author (D. Krupka) acknowledges the support of the National Science Foundation of China (Grant no. 10932002) and the Czech Science Foundation (Grant no. 201/09/0981).

\section{References}

[1] D. Gromoll, W. Klingenberg, and W. Meyer, Riemannsche Geometrie im Grossen, Lecture Notes in Mathematics, no. 55, Springer, Berlin, Germany, 1968.

[2] Z. Shen, Differential Geometry of Spray and Finsler Spaces, Kluwer Academic Publishers, Dordrecht, The Netherlands, 2001.

[3] O. Krupková and G. E. Prince, "Second order ordinary differential equations in jet bundles and the inverse problem of the calculus of variations," in Handbook of Global Analysis, D. Krupka and D. Saunders, Eds., pp. 837-904, Elsevier Sci. B. V., Amsterdam, The Netherlands, 2008.

[4] S. Lang, Fundamentals of Differential Geometry, vol. 191 of Graduate Texts in Mathematics, Springer, New York, NY, USA, 1999.

[5] R. S. Palais and C. L. Terng, "Natural bundles have finite order," Topology, vol. 19, no. 3, pp. 271-277, 1977.

[6] D. J. Saunders, "Jet fields, connections and second-order differential equations," Journal of Physics A, vol. 20, no. 11, pp. 32613270, 1987.

[7] S. Sternberg, Lectures on Differential Geometry, Prentice-Hall, Englewood Cliffs, NJ, USA, 1964.

[8] H. Goldschmidt and S. Sternberg, "The Hamilton-Cartan formalism in the calculus of variations," Annales de l'Institut Fourier, vol. 23, no. 1, pp. 203-267, 1973.

[9] D. Krupka, "Some geometric aspects of variational problems in fibred manifolds," Folia Facultatis Scientiarum Naturalium Universitatis Purkynianae. Brunensis, vol. 14, pp. 1-65, 1973.

[10] R. Abraham and J. E. Marsden, Foundations of Mechanics, Benjamin/Cummings, Reading, Mass, USA, 2nd edition, 1978.

[11] D. Krupka, O. Krupková, G. Prince, and W. Sarlet, "Contact symmetries of the Helmholtz form," Differential Geometry and Its Applications, vol. 25, no. 5, pp. 518-542, 2007.

[12] W. Sarlet, A. Vandecasteele, F. Cantrijn, and E. Martínez, "Derivations of forms along a map: the framework for timedependent second-order equations," Differential Geometry and Its Applications, vol. 5, no. 2, pp. 171-203, 1995.

[13] D. Krupka and J. Janyška, Lectures on Differential Invariants, vol. 1 of Folia Facultatis Scientiarum Naturalium Universitatis Purkynianae Brunensis. Mathematica, University J. E. Purkyně, Brno, Czech Republic, 1990.

[14] D. R. Grigore and D. Krupka, "Invariants of velocities and higher-order Grassmann bundles," Journal of Geometry and Physics, vol. 24, no. 3, pp. 244-264, 1998.

[15] I. Kolář, P. W. Michor, and J. Slovák, Natural Operations in Differential Geometry, Springer, Berlin, Germany, 1993.

[16] D. Krupka and M. Krupka, "Jets and contact elements," in Proceedings of the Seminar on Differential Geometry (Opava, 2000), vol. 2 of Mathematical Publications, pp. 39-85, Silesian Univ. Opava, Opava, Czech Republic, 2000.

[17] W. Sarlet and F. Cantrijn, "Special symmetries for Lagrangian systems and their analogues in nonconservative mechanics," in Proceedings of the Conference on Differential Geometry and Its Applications, D. Krupka, Ed., pp. 247-260, Univ. J. E. Purkyně, Brno, Czech Republic, 1984.
[18] D. Krupka, "Local invariants of a linear connection," in Differential Geometry (Budapest, 1979), vol. 31 of Colloq. Math. Soc. János Bolyai, pp. 349-369, North-Holland, Amsterdam, The Netherlands, 1982. 


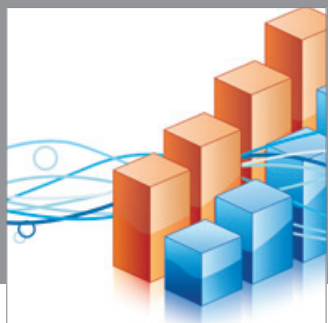

Advances in

Operations Research

mansans

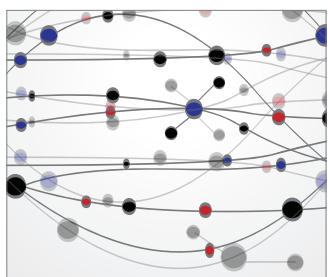

The Scientific World Journal
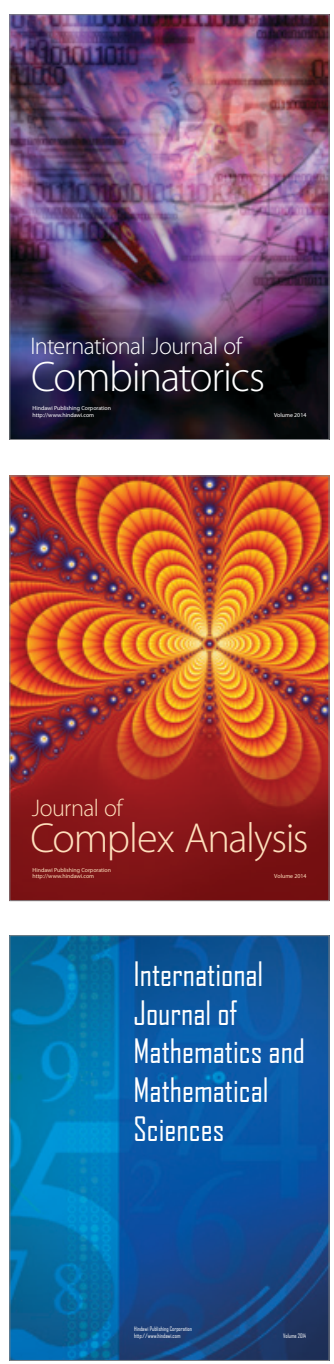
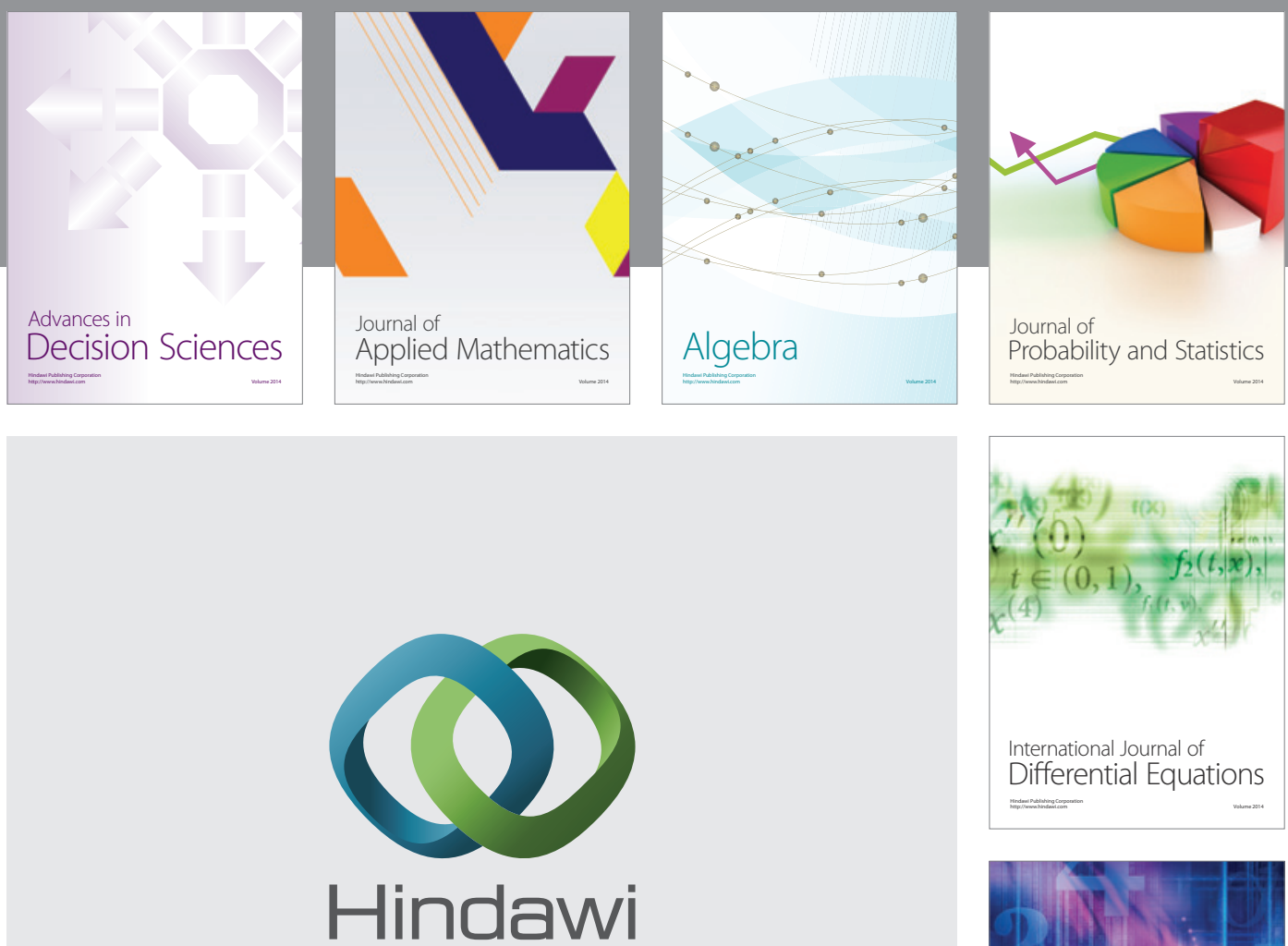

Submit your manuscripts at http://www.hindawi.com
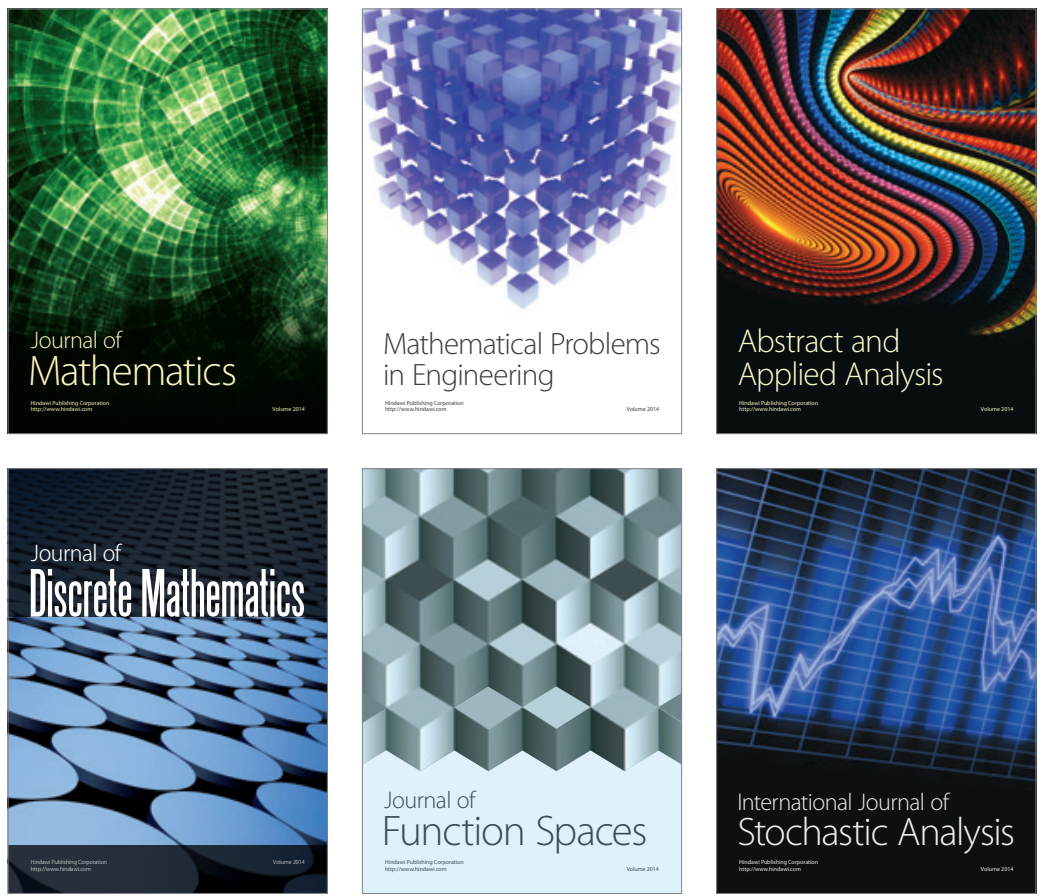

Journal of

Function Spaces

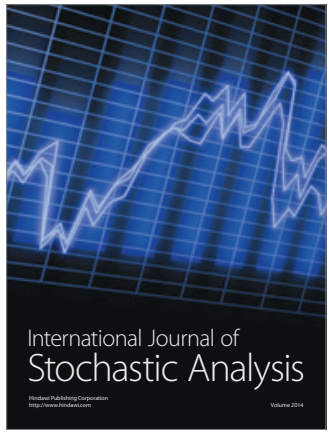

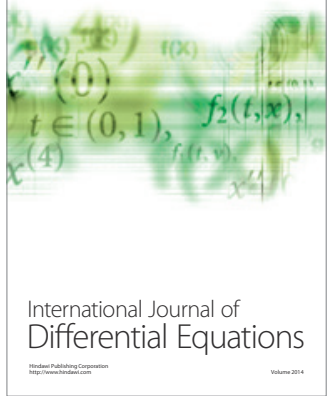
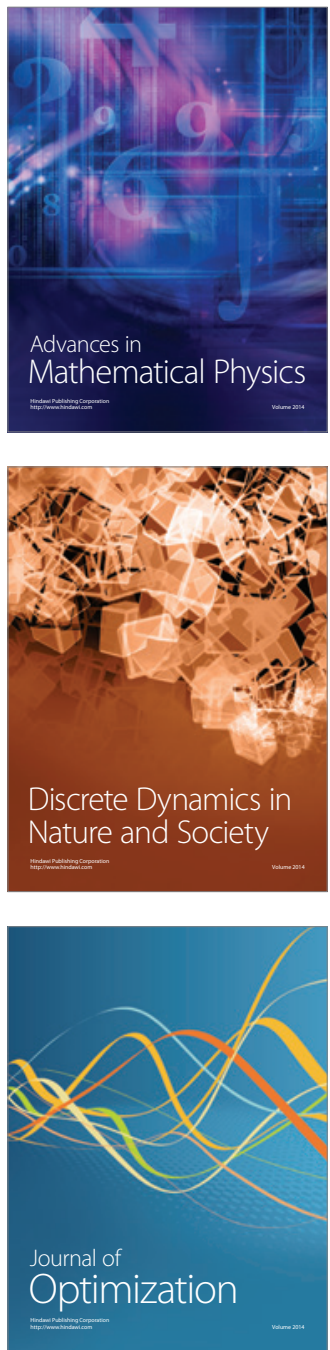